\title{
Persistent Cholestasis After Critical Illness
}

\author{
Than Aung ${ }^{\mathrm{a}, \mathrm{b}}$, Chaw Su Naing ${ }^{\mathrm{a}}$
}

\begin{abstract}
Sclerosing cholangitis in critically ill patients (SC-CIP) is a new form of secondary sclerosing cholangitis associated with rapid progression to liver cirrhosis. We report a case of 60 -year-old female with chronic cholestasis after receiving treatment in intensive care. Our purpose was to increase awareness and timely recognize the diagnosis of SCCIP.
\end{abstract}

Keywords: Sclerosing cholangitis in critically ill patient; Cirrhosis

\section{Introduction}

Secondary sclerosing cholangitis (SSC) is a rare form of chronic cholestatic liver disease with inflammatory and fibrotic changes of the biliary tract leading to biliary cirrhosis. We present a case of SSC developing after a course of critical illness.

\section{Case Report}

A 60-year-old female with history of diabetes and hypertension came to the hospital for sudden onset of right upper abdominal pain associated with vomiting for 1 day duration. In emergency unit, her vital signs were stable (temperature $35.6^{\circ} \mathrm{C}$, blood pressure 100/51 $\mathrm{mm} \mathrm{Hg}$, heart rate 73 beat per minute regular, and pulse oximetry $97 \%$ room air). On examination, there was guarding, tenderness in epigastrium and negative Murphy sign. Laboratory results showed elevated white blood cell, amylase, lipase, and transaminase (amylase 2,356 U/L (normal 33 - $126 \mathrm{U} / \mathrm{L}$ ), lipase $400 \mathrm{U} / \mathrm{L}$ (normal 14 - $40 \mathrm{U} / \mathrm{L}$ ), lactate dehydrogenase 1,037 U/L (normal $180-380 \mathrm{U} / \mathrm{L}$ ), bilirubin $43 \mu \mathrm{mol} / \mathrm{L}$ (normal $3-24 \mu \mathrm{mol} / \mathrm{L}$ ), alanine aminotransferase $99 \mathrm{U} / \mathrm{L}$ (normal 7 - $36 \mathrm{U} / \mathrm{L}$ ), aspartate transaminase $228 \mathrm{U} / \mathrm{L}$ (normal 15 - $33 \mathrm{U} / \mathrm{L}$ ), alkaline phosphatase $40 \mathrm{U} / \mathrm{L}$ (normal 32

Manuscript accepted for publication August 10, 2016

aDepartment of Internal Medicine, Singapore General Hospital, Outram Road, Singapore 169608, Singapore

${ }^{b}$ Corresponding Author: Than Aung, Department of Internal Medicine, Singapore General Hospital, Outram Road, Singapore 169608, Singapore.

Email: than.aung@singhealth.com.sg

doi: http://dx.doi.org/10.14740/jmc2603w
- $103 \mathrm{U} / \mathrm{L}$ ), and white blood cell $11.6 \times 10^{9} / \mathrm{L}$ (normal $4-10 \times$ $10^{9} / \mathrm{L}$ with neutrophilia $\left.80 \%\right)$ ). Chest $\mathrm{X}$-ray and abdomen $\mathrm{X}$ ray were unremarkable.

She was managed as acute pancreatitis secondary to gall stones, by starting with broad-spectrum intravenous antibiotics, normal saline infusion. While waiting for urgent $\mathrm{CT}$ of abdomen, she became hemodynamically unstable and developed septic shock and lactic acidosis (temperature $38^{\circ} \mathrm{C}$, pulse oximetry $88 \%$, blood pressure $90 / 50 \mathrm{~mm} \mathrm{Hg}$, heart rate 114 beat per minute, respiratory rate 30 per minute, arterial blood gas $\mathrm{pH} 7.3$ (7.35 - 7.45), $\mathrm{PO}_{2} 62 \mathrm{~mm} \mathrm{Hg}(75$ - 100), bicarbonate $19 \mathrm{mmol} / \mathrm{L}(21-27)$, and lactate $5.1 \mathrm{mmol} / \mathrm{L}(0.5-2.2))$. She was immediately transferred to intensive care unit (ICU) for ventilation and inotropic support with dobutamine and norepinephrine.

Finding of acute pancreatitis, gangrenous cholecystitis with stone on $\mathrm{CT}$ of abdomen prompted her to undergo urgent open cholecystectomy. After surgery in ICU, patient remained hypotensive requiring three inotropes to sustain the mean arterial pressure above $65 \mathrm{~mm} \mathrm{Hg}$ with dobutamine, norepinephrine and vasopressin for 4 days, then dobutamine and norepinephrine for another 6 days, subsequently dobutamine alone for 2 days and mechanical ventilation (with positive end expiratory pressure (PEEP) $5-12 \mathrm{~cm} \mathrm{H}_{2} \mathrm{O}$ ). Later, culture of bile grew Escherichia coli and Klebsiella and antibiotics was tailored based on sensitivity. Her condition was complicated with acute kidney injury, non-ST elevation myocardial infarction (peak troponin T 4.9 UG/L $(<0.03)$ ) secondary to systemic hypoperfusion. After having continuous renal replacement therapy for a week, her renal function slowly improved and was nearly back to normal upon discharge. The patient underwent echocardiogram to estimate the heart function which reported left ventricular ejection fraction of $24 \%$ with regional wall motion abnormality. Myocardial infarction was treated conservatively. Patient stayed in hospital for a month and was subsequently discharged. She had raised alkaline phosphatase $292 \mathrm{U} / \mathrm{L}$ and mild elevation of bilirubin $32 \mu \mathrm{mol} / \mathrm{L}$ on discharge. Hepatitis $\mathrm{B}$ and $\mathrm{C}$ and human immunodeficiency virus serology were negative.

Two weeks later, she was admitted for fever and jaundice. She was alert, looked non-toxic, and examinations were unremarkable. Bilirubin was elevated to $166 \mu \mathrm{mol} / \mathrm{L}$ and alkaline phosphatase was steady at $267 \mathrm{U} / \mathrm{L}$, gamma glutamyl transferase (GGT) was $468 \mathrm{U} / \mathrm{L}$ (normal 7 - $39 \mathrm{U} / \mathrm{L}$ ), and white blood cell was $14.3 \times 10^{9} / \mathrm{L}$. Anti-liver antibodies profile, autoimmune workup, and blood cultures turned out negative. Being suspected of having cholangitis, she was treated with intravenous broad-spectrum antibiotics. CT of abdomen showed 


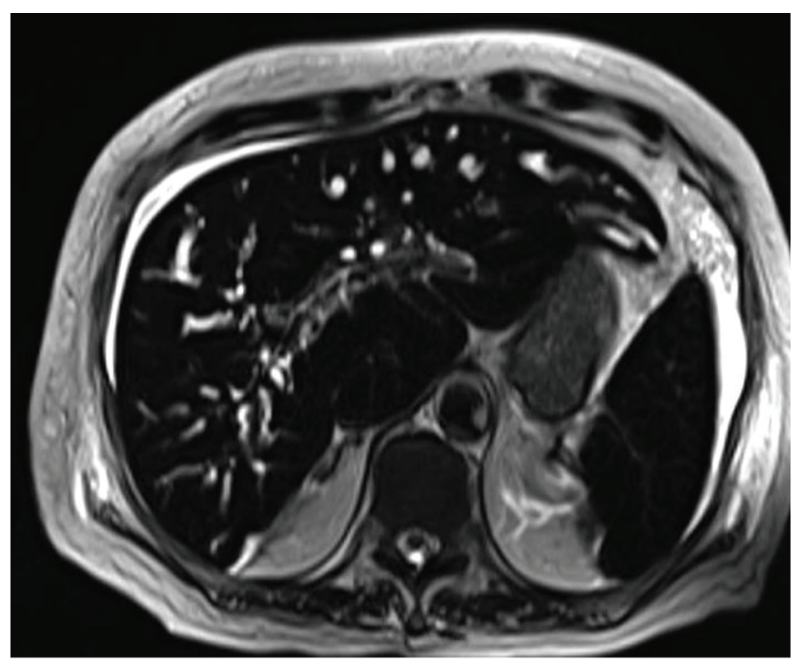

Figure 1. Magnetic resonance cholangiography finding of SC-CIP: alternating biliary strictures and dilatation.

collections around the pancreas secondary to prior pancreatitis. There was mildly dilated intrahepatic bile ducts and prominent extrahepatic bile duct with no choledocholithiasis. Her serum bilirubin and alkaline phosphatase remained static, leading her to further evaluation with magnetic resonance cholangiography (MRCP) for assessment of biliary system. It revealed cholangitis, pseudocysts of pancreas, and two small liver abscesses. Despite treating with antibiotics for a week, the surge in alkaline phosphate to $332 \mathrm{U} / \mathrm{L}$ prompted her to undergo endoscopic ultrasound (EUS) for evaluation of bile ducts. There was sludge in proximal common bile duct (CBD). Patient was clinically better. Her bilirubin and alkaline phosphatase remained stable. She was given close follow-up in clinic.

A month later, patient was seen for worsening of jaundice (bilirubin $296 \mu \mathrm{mol} / \mathrm{L}$ and alkaline phosphatase $426 \mathrm{U} / \mathrm{L}$ ). She was asymptomatic. Extensive infectious workup such as hepatitis A, B, C, E, cytomegalovirus, herpes simplex virus, and Epstein-Barr virus serology was negative. Repeated MRCP showed persisting cholangitis and there were no stones or acute pancreatitis. Bilirubin and alkaline phosphatase trended down to $189 \mu \mathrm{mol} / \mathrm{L}$ and $286 \mathrm{U} / \mathrm{L}$, respectively after starting ursodeoxycholic acid.

Four months later, left ventricular ejection improved to $40 \%$ on repeated echocardiography for recent myocardial infarction. The risk was relatively lower for the patient to undergo invasive procedure such as ERCP for evaluation of persistent cholestatic liver function (bilirubin $177-229 \mu \mathrm{mol} / \mathrm{L}$ and alkaline phosphatase 221 - $268 \mathrm{U} / \mathrm{L}$ ). ERCP disclosed biliary sludge in CBD. There was no CBD or intrahepatic ductal dilatation. The biliary stent was deployed in CBD.

During the biliary stent removal after 2 months of insertion, finding of underfilling of intrahepatic duct on cholangiogram prompted her to repeat MRCP which discovered dilatation, beading, strictures of intrahepatic ducts bilaterally (Figs. 1 and 2).

Given the prior prolonged critical illness, persistent cholestasis with no previous preexisting hepatobiliary condition, the findings on imaging were consistent with SSC related

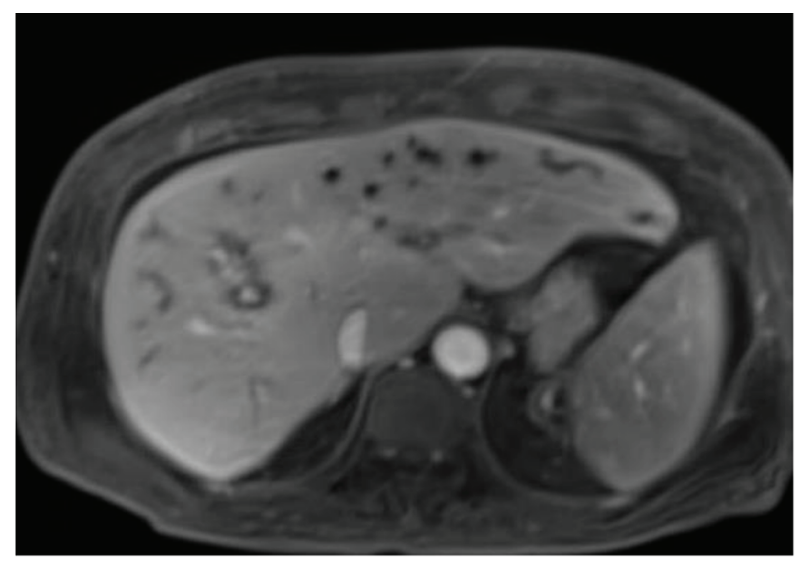

Figure 2. Intrahepatic ducts dilatations and strictures.

to critical illness.

\section{Discussion}

SSC is a chronic cholestatic biliary disease with inflammation, fibrosis and destruction of bile ducts leading to biliary cirrhosis [1].

A new form of SSC, sclerosing cholangitis in critically ill patients (SC-CIP) characterized by obstruction of bile ducts after having treatment in ICU, is associated with rapid progression to liver cirrhosis. The patients with SC-CIP generally do not have a history of preceding biliary or liver disease.

It is a rare biliary disease and there are insufficient data to determine the true prevalence of SC-CIP since it is relatively new and underrecognized biliary disorder.

The speculated risk factors contributing to the development of SC-CIP are severe hypotension with decrease in mean arterial blood pressure (MAP) to $<65 \mathrm{~mm} \mathrm{Hg}$, mechanical ventilation with high PEEP, microcirculatory disturbances, systemic inflammatory response syndrome (SIRS), and prone positioning.

The severe hypotension appears to be the most significant trigger in the pathogenesis of SC-CIP. The biliary epithelium receives blood supply exclusively from peribiliary plexus of the hepatic artery, in contrast to the hepatic parenchyma which has a dual blood supply from the hepatic artery and the portal vein. Therefore, the biliary epithelium is more susceptible to ischemia than hepatocytes $[2,3]$.

Decreased MAP, the use of catecholamines, mechanical ventilation with high PEEP and disturbances of the microcirculation compromise the blood supply to bile ducts [4-7]. Hypotension causes direct ischemic injury to bile ducts. Using inotrope to sustain the MAP does not parallel with improved hepatosplanchnic perfusion. Norepinephrine has vaso-constrictive properties, and can cause decreased splanchnic blood flow. Mechanical ventilation with high PEEP to maintain or restore the oxygenation has negative effects on the microcirculation in the gastrointestinal tract. Keeping patient in ICU prone position has synergistic effect on PEEP and may increase the amount of well aerated lung tissue but it has been postulated 
prone positioning increases intra-abdominal pressure which may compromise hepatosplanchnic blood flow.

Microcirculatory disturbances involving the peribiliary plexus are also one of the triggering factors of developing SCCIP. Extensive tissue damage caused by surgery can initiate hypercoagulable state which can contribute to occlusion of the peribiliary plexus.

SC-CIP presents with rapidly increasing serum alkaline phosphatase and gamma-glutamyltransferase with bilirubin. Clinical signs are non-specific and cholestatic derangement of liver function persists despite recovery from the primary disease that differentiates patients with SC-CIP from patients with endotoxin-associated jaundice, which resolves after clinical recovery $[3,5]$. Imaging or endoscopic retrograde cholangiography (ERCP) diagnoses biliary casts and detects the biliary stricture formation.

Biliary cast formation was seen in majority of SC-CIP. Biliary cast formation in non-transplanted livers is rare. Many factors, such as biliary infection, bile duct damage, biliary ischemia, and hemolysis play roles in cast formation [8].

The histological features of SC-CIP are initially nonspecific. After a few months, there is a progressive fibrosis of the intrahepatic bile ducts with bile duct proliferation, portal inflammatory infiltrates, periductal fibrosis, and canalicular cholestasis $[3,4,7]$.

ERCP is a standard test to diagnose biliary casts or sludge and detect biliary stricture formation. Intrahepatic biliary cast formation is the earliest endoscopic finding [4, 5, 7, 9]. Imaging with MRCP can identify strictures, dilatation, beading, wall thickening of intrahepatic bile ducts and microabscesses.

Our patient had combination of all above mentioned risk factors for ischemic injury to bile ducts such as hypotension with increasing requirement of inotropes, mechanical ventilator with PEEP setting, urgent laparotomy complicated with multi-organ dysfunction together with clinical presentation of progressive persistent cholestasis and imaging evidence of biliary sludge, strictures, dilatation and beading which are compatible with SC-CIP.

Treatment options of SC-CIP are limited. Endoscopic removal of intraductal biliary cast promotes the bile flow and giving ursodeoxycholic acid facilitates biochemical improvement in cholestasis. Liver transplant is the only definitive treatment for most advanced cases and all patients should be evaluated for transplant $[5,7]$.

Imaging on subsequent follow-up of our patient revealed liver cirrhosis. The patient underwent evaluation for liver transplant and has been closely monitored on following visits.

\section{Conclusion}

SC-CIP is a severe progressive complication of patients in ICU with rapid progression to cirrhosis. Being a relatively new entity with non-specific clinical features, diagnosis of SC-CIP may be overlooked. SC-CIP can be detected by raising the clinical index of suspicion in patient with unexplained cholestasis who had sepsis, long-term ICU therapy. Because of its serious complications and poor prognosis, further studies are required for prevention and new treatment options of SC-CIP.

\section{Grant}

This research received no specific grant from any funding agency.

\section{Conflicts of Interest}

None.

\section{References}

1. Imam MH, Talwalkar JA, Lindor KD. Secondary sclerosing cholangitis: pathogenesis, diagnosis, and management. Clin Liver Dis. 2013;17(2):269-277.

2. Abdalian R, Heathcote EJ. Sclerosing cholangitis: a focus on secondary causes. Hepatology. 2006;44(5):10631074.

3. Ruemmele P, Hofstaedter F, Gelbmann CM. Secondary sclerosing cholangitis. Nat Rev Gastroenterol Hepatol. 2009;6(5):287-295.

4. Gelbmann CM, Rummele P, Wimmer M, Hofstadter F, Gohlmann B, Endlicher E, Kullmann F, et al. Ischemiclike cholangiopathy with secondary sclerosing cholangitis in critically ill patients. Am J Gastroenterol. 2007;102(6):1221-1229.

5. Lin T, Qu K, Xu X, Tian M, Gao J, Zhang C, Di Y, et al. Sclerosing cholangitis in critically ill patients: an important and easily ignored problem based on a German experience. Front Med. 2014;8(1):118-126.

6. Leonhardt S, Veltzke-Schlieker W, Adler A, Schott E, Hetzer R, Schaffartzik W, Tryba M, et al. Trigger mechanisms of secondary sclerosing cholangitis in critically ill patients. Crit Care. 2015;19:131.

7. Kirchner GI, Rummele P. Update on Sclerosing Cholangitis in Critically Ill Patients. Viszeralmedizin. 2015;31(3):178-184

8. Parry SD, Muiesan P. Cholangiopathy and the biliary cast syndrome. Eur J Gastroenterol Hepatol. 2003;15(4):341343.

9. Pohl J, Mayer G. Secondary Sclerosing Cholangitis. Video Journal and Encyclopedia of GI Endoscopy. 2013. 